\title{
Measuring the food environment using geographical information systems: a methodological review
}

\author{
Hélène Charreire ${ }^{1}$, Romain Casey ${ }^{2}$, Paul Salze ${ }^{3}$, Chantal Simon $^{2}$, Basile Chaix ${ }^{4}$, \\ Arnaud Banos ${ }^{3}$, Dominique Badariotti ${ }^{3}$, Christiane Weber ${ }^{3}$ and Jean-Michel Oppert ${ }^{1,5, *}$ \\ 'UMR INSERM U 557/INRA U 11 25/CNAM, University Paris 13, CRNH IdF, Bobigny, France: ${ }^{2}$ INSERM U \\ 870/INRA U 1235, Human Nutrition Research Center (CRNH Rhône-Alpes), University of Lyon, Hospices Civils \\ de Lyon, Oullins, France: ${ }^{3}$ ERL 7230, CNRS, Image, Ville, Environnement, University of Strasbourg, Strasbourg, \\ France: ${ }^{4}$ INSERM U 707, Paris, France: ${ }^{5}$ Service de Nutrition GH Pitié-Salpêtrière (AP-HP), University Pierre et \\ Marie Curie-Paris, CRNH IdF, Paris, France
}

Submitted 24 May 2009: Accepted 28 February 2010: First published online 21 April 2010

\begin{abstract}
Objective: Through a literature review, we investigated the geographic information systems (GIS) methods used to define the food environment and the types of spatial measurements they generate.

Design: Review study.

Setting: Searches were conducted in health science databases, including Medline/ Pubmed, PsycINFO, Francis and GeoBase. We included studies using GIS-based measures of the food environment published up to 1 June 2008.

Results: Twenty-nine papers were included. Two different spatial approaches were identified. The density approach quantifies the availability of food outlets using the buffer method, kernel density estimation or spatial clustering. The proximity approach assesses the distance to food outlets by measuring distances or travel times. GIS network analysis tools enable the modelling of travel time between referent addresses (home) and food outlets for a given transportation network and mode, and the assumption of travel routing behaviours. Numerous studies combined both approaches to compare food outlet spatial accessibility between different types of neighbourhoods or to investigate relationships between characteristics of the food environment and individual food behaviour. Conclusions: GIS methods provide new approaches for assessing the food environment by modelling spatial accessibility to food outlets. On the basis of the available literature, it appears that only some GIS methods have been used, while other GIS methods combining availability and proximity, such as spatial interaction models, have not yet been applied to this field. Future research would also benefit from a combination of GIS methods with survey approaches to describe both spatial and social food outlet accessibility as important determinants of individual food behaviours.
\end{abstract}

\section{Background}

Food intake is considered a complex behaviour of multifactorial origin ${ }^{(1)}$. A socio-ecological approach to understanding such behaviour is recognised as being a useful framework for integrating the numerous influences present at both the individual and environmental levels ${ }^{(1-5)}$. There is growing interest in the environmental context as related to food behaviour; this includes both the social and physical environment. In this relatively recent field of research, Glanz et $a l^{(6,7)}$ identified different aspects of the food environment. These include the 'community nutritional environment' defined as the number, type, location and accessibility of food outlets, and the 'consumer nutritional environment' defined by what consumers encounter in and around food outlets (prices, promotions, nutritional quality). In terms of community nutrition, a number of studies and reviews emphasise the influence of spatial accessibility of food upon the relationship between food environment, individual food choice and, ultimately, risk of chronic diseases such as obesity $^{(3,6,8-12)}$. An important research issue lies in identifying and describing the different methodological procedures that can be used to specifically assess the spatial accessibility of food outlets.

Various methods, both objective and subjective, have been used to assess variables related to the presence and type of food outlet. Subjective methods include surveys of individual perception of food outlets available to 
neighbourhood residents ${ }^{(13,14)}$. Among objective methods, geographic measures are most frequently used to assess the food environment ${ }^{(15)}$. Some of these are provided by spatial analysis methods based on geographic information systems (GIS). GIS are computer-based methods and tools, which, via different information sources, enable spatial and thematic data to be organised, managed and combined, and results to be represented and analysed according to geographic location $^{(16)}$. Analyses can then be carried out to localise and model potential spatial interactions between the different types of information at hand.

In public health, examples of the use of GIS methods include the analysis of disparities in access to healthcare $^{(17)}$ and, more recently, the association between built environment and physical activity ${ }^{(6,18)}$. Application of GIS to the food environment is relatively new in public health nutrition. Use of a geographic model of analysis may help to identify spatial inequalities in access to food outlets, and in turn, influence policies and incite urban planners to modify the food environment accordingly. In this context, a major challenge lies in ensuring appropriate and effective use of GIS data and spatial analysis methods to measure the food environment ${ }^{(19,20)}$. Despite the growing use of GIS, we were unable to find a literature review of GIS methods used to assess the food environment. The aim of the present methodological article was to describe GIS methods already in use in this field and to discuss their relevance for increasing our understanding of food environment attributes.

\section{Methods}

We sought to identify all studies that used GIS to measure the proximity and/or density of food outlets so as to characterise attributes of the food environment. A search of the literature was conducted with the OVID interface in the following social and health science databases: Medline/ Pubmed, PsycINFO, Francis and GeoBase. The search was conducted using different combinations of keywords (in the title or abstract) such as 'food environment', 'food outlets', 'access', 'availability' and 'geographic information system'. The search was restricted to human populations, and studies on both adults and children were included. In addition, the reference sections of the articles included were reviewed. The search was restricted to English language articles published between January 1999 and June 2008.

The main inclusion criterion in the review was the use of GIS-based techniques of spatial analysis to measure the food environment. We excluded studies that relied only on survey participants to characterise the food environment $^{(13)}$ and articles that used GIS only as a geocoding tool (process assigning geographic coordinates to a point, e.g. street addresses to food outlet) to map various data ${ }^{(21)}$. Data extracted in addition to GIS methods were: study location, scale (e.g. census tract), food environment outcome (e.g. supermarkets, fast food), covariates and main findings.

\section{Results}

\section{Study characteristics}

An initial search of online databases and of the reference sections of the articles included identified 1070 papers. After preliminary screening based on the title or abstract, thirty-eight full-text papers were retrieved for further assessment. In the final step, twenty-nine articles with GIS-based measurements of the food environment were included in the review. Table 1 summarises data extracted for each paper. Seventeen reviewed studies were conducted in the United States, four in Australia, three in Canada, three in New Zealand and two in England. The selected studies fell into two categories: (i) studies that explore the relationships between characteristics of the food environment and measurements of individual food behaviours; and (ii) studies that compare accessibility of food outlets in different types of neighbourhoods.

\section{Relationship between food environment and individual food behaviour}

Among the twenty-nine articles reviewed, eleven (38\%) analysed associations between food environment and individual food behaviours ${ }^{(22-26)}$, weight status ${ }^{(27-31)}$ or perceived availability of healthy food ${ }^{(32)}$. In those studies, the addresses of respondents were geocoded and used as references for GIS analyses. Four studies were performed on children or teenagers ${ }^{(24,27,28,31)}$, and the others on adult populations (one specifically concerned pregnant women ${ }^{(33)}$ ). The outcomes of selected studies were consumption of fruits and vegetables ${ }^{(22-24,28)}$, perception of availability of healthy food ${ }^{(32)}$, dietary patterns ${ }^{(26,33)}$ and prevalence of overweight or obesity ${ }^{(27,29-31)}$. In most data sets (seven out of eleven), individual characteristics were collected from the year $2000^{(22-24,26,28,31,32)}$. In three studies, the date on which food outlet lists were drawn up was not mentioned ${ }^{(28-30)}$. In the other studies, the date given for the food outlet list corresponded to the date of collection of individual data ( \pm 2 years).

The covariates most frequently used in the analyses included individual demographic and socio-economic characteristics, and individual behaviour such as smoking and physical activity or sedentary behaviour (Table 1).

\footnotetext{
Spatial access to food outlets according to the type of neighbourbood

The aim of most articles retrieved (eighteen out of twentynine; $62 \%$ ) was to assess and compare neighbourhoods according to spatial access to food outlets. All these articles considered the neighbourhood as the area of study. However, the scale of the neighbourhoods varied: it
} 
Table 1 Summary descriptive table of the studies included in the review

\begin{tabular}{|c|c|c|c|c|c|}
\hline Author(s) (year) & $\begin{array}{l}\text { Location } \\
\text { Scale/population }\end{array}$ & $\begin{array}{l}\text { Measures food } \\
\text { availability }\end{array}$ & GIS method & Other variables & Findings \\
\hline $\begin{array}{l}\text { Apparicio et al. } \\
(2007)^{(39)}\end{array}$ & $\begin{array}{l}\text { Montreal (Canada) } \\
\text { Census tract block }\end{array}$ & $\begin{array}{l}\text { Supermarket: } \\
\text { - Proximity } \\
\text { - Diversity } \\
\text { - In the immediate } \\
\quad \text { surrounding }\end{array}$ & $\begin{array}{l}\text { - Euclidean distance to the } \\
\text { closest supermarket } \\
\text { - Number of supermarkets at a } \\
\text { distance less than } 1000 \mathrm{~m} \\
\text { - Mean distance to the three } \\
\text { closest supermarkets to } \\
\text { different companies }\end{array}$ & $\begin{array}{l}\text { Deprivation index } \\
\text { - Income } \\
\text { - Lone-parent families } \\
\text { - Unemployment } \\
\text { - Education } \\
\text { - Recent immigrants }\end{array}$ & $\begin{array}{l}\text { Food deserts do not represent a major } \\
\text { problem in Montreal }\end{array}$ \\
\hline $\begin{array}{l}\text { Austin et al. } \\
\qquad(2005)^{(44)}\end{array}$ & $\begin{array}{l}\text { Chicago (Illinois) } \\
\text { Census tract } \\
\text { School addresses }\end{array}$ & $\begin{array}{l}\text { Fast food: } \\
\text { - Density } \\
\text { - Proximity to school }\end{array}$ & $\begin{array}{l}\text { - Buffer }(400 / 800 \mathrm{~m} \text { around } \\
\text { school) } \\
\text { - Euclidean distance to school } \\
\text { from the closest fast food }\end{array}$ & $\begin{array}{l}\text { Contextual data } \\
\text { - Level of commercialization } \\
\text { (percentage of commercial } \\
\text { land) } \\
\text { - Household income } \\
\text { - Inside or outside downtown }\end{array}$ & $\begin{array}{l}\text { Fast foods are concentrated within short } \\
\text { distance from schools }\end{array}$ \\
\hline $\begin{array}{l}\text { Baker et al. } \\
\quad(2006)^{(48)}\end{array}$ & $\begin{array}{l}\text { St Louis (Missouri) } \\
\text { Census tract }\end{array}$ & $\begin{array}{l}\text { Clustering to: } \\
\text { - Supermarket } \\
\text { - Fast food }\end{array}$ & $\begin{array}{l}\text { Spatial clustering } \\
\text { - Circular window } \\
\text { - Number of food outlets }\end{array}$ & $\begin{array}{l}\text { - Composite score of availability } \\
\text { to supermarket and fast food } \\
\text { - Contextual data: race, income } \\
\text { (level of poverty) }\end{array}$ & $\begin{array}{l}\text { Income and race seem to be associated } \\
\text { not only with the location of food outlet } \\
\text { but also with the selection of food } \\
\text { available }\end{array}$ \\
\hline $\begin{array}{l}\text { Block \& Kouba } \\
(2006)^{(49)}\end{array}$ & $\begin{array}{l}\text { Chicago (Illinois), two } \\
\text { neighbouring } \\
\text { communities (Austin \& } \\
\text { Oak Park) }\end{array}$ & $\begin{array}{l}\text { Proximity to: } \\
\text { - Supermarket } \\
\text { - Independant grocery } \\
\text { - Convenience store } \\
\text { - Other food outlets }\end{array}$ & $\begin{array}{l}\text { - Buffer }(0 \cdot 25 / 0 \cdot 5 / 0 \cdot 75 / 1 \text { mile } \\
\text { and }>1 \text { mile around food store })\end{array}$ & $\begin{array}{l}\text { - Food survey: cost and quality of } \\
\text { produce } \\
\text { - Contextual data: households } \\
\text { having no car }\end{array}$ & $\begin{array}{l}\text { Type and number of grocery stores differ } \\
\text { between Austin and Oak Park }\end{array}$ \\
\hline $\begin{array}{l}\text { Block et al. } \\
(2004)^{(45)}\end{array}$ & $\begin{array}{l}\text { New Orleans (Louisiana) } \\
\text { Census tract }\end{array}$ & $\begin{array}{l}\text { Fast food: } \\
\text { - Density } \\
\text { - Distance }\end{array}$ & $\begin{array}{l}\text { Buffer (shopping area) }(0.5 / 1 \\
\text { mile around census tract) }\end{array}$ & $\begin{array}{l}\text { Contextual data: } \\
\text { - Black residents } \\
\text { - Low income } \\
\text { Ajusted by: } \\
\text { - Alcohol outlet density } \\
\text { - Highways } \\
\text { - Median home value }\end{array}$ & $\begin{array}{l}\text { Fast food outlets are geographically } \\
\text { associated with predominantly black } \\
\text { and low-income neighbourhoods }\end{array}$ \\
\hline $\begin{array}{l}\text { Bodor et al. } \\
\quad(2008)^{(22)}\end{array}$ & $\begin{array}{l}\text { New Orleans (Louisiana), } \\
\text { older urban area } \\
102 \text { households (16 years } \\
\text { and more) }\end{array}$ & $\begin{array}{l}\text { Distance to and } \\
\text { density of: } \\
\text { - Supermarket } \\
\text { - Small food store }\end{array}$ & $\begin{array}{l}\text { - Buffer }(100 \mathrm{~m} / 1 \mathrm{~km} \text { around } \\
\text { home) } \\
\text { - Density of food store } \\
\text { - Euclidean distance: to the } \\
\text { closest small food store, to } \\
\text { the closest supermarket }\end{array}$ & $\begin{array}{l}\text { - Individual level fruit and } \\
\text { vegetable intake and SES } \\
\text { characteristics } \\
\text { - In-store level availability of: } \\
\text { fresh, canned and frozen fruit } \\
\text { and vegetables }\end{array}$ & $\begin{array}{l}\text { - Access to a small food store within } \\
100 \mathrm{~m} \text { of home was marginally asso- } \\
\text { ciated with an increased fruit intake } \\
\text { - No association was found between } \\
\text { intake and access to supermarkets }\end{array}$ \\
\hline
\end{tabular}


Table 1 Continued

\begin{tabular}{|c|c|c|c|c|c|}
\hline Author(s) (year) & $\begin{array}{l}\text { Location } \\
\text { Scale/population }\end{array}$ & $\begin{array}{l}\text { Measures food } \\
\text { availability }\end{array}$ & GIS method & Other variables & Findings \\
\hline $\begin{array}{l}\text { Burdette et al. } \\
\quad(2004)^{(27)}\end{array}$ & $\begin{array}{l}\text { Cincinnati (Ohio) } \\
7020 \text { children ( } 3-4 \text { years) }\end{array}$ & $\begin{array}{l}\text { Distance to: } \\
\text { - Playgrounds } \\
\text { - Fast food restaurants }\end{array}$ & $\begin{array}{l}\text { Distance by street travel } \\
\text { between child's home and the } \\
\text { nearest playground and fast } \\
\text { food }\end{array}$ & $\begin{array}{l}\text { Individual variables } \\
\text { - Age, sex, race, income, BMI } \\
\text { - Household size } \\
\text { Neighbourhood safety: } \\
\text { - Number of serious crime } \\
\text { - Number of emergency police } \\
\text { calls (911) }\end{array}$ & $\begin{array}{l}\text { No association between } \\
\text { - Overweight and neighbourhood safety } \\
\text { - Overweight and proximity to play- } \\
\text { grounds or fast foods }\end{array}$ \\
\hline $\begin{array}{l}\text { Burns \& Inglis } \\
\qquad(2007)^{(34)}\end{array}$ & $\begin{array}{l}\text { City of Casey (Melbourne, } \\
\text { Australia) } \\
\text { Collection districts }\end{array}$ & $\begin{array}{l}\text { Travel time of: } \\
\text { - Supermarket } \\
\text { - Fast food by car and } \\
\text { by bus }\end{array}$ & $\begin{array}{l}\text { Modelling travel time } \\
\text { depending on: } \\
\text { - Type of road (speed limit) } \\
\text { - Frequency of buses }\end{array}$ & $\begin{array}{l}\text { - Population density } \\
\text { - Deprivation index: SEIFA }\end{array}$ & $\begin{array}{l}\text { - Less advantaged areas had closer } \\
\text { access to fast foods } \\
\text { - More advantaged areas had closer } \\
\text { access to a major supermarket }\end{array}$ \\
\hline $\begin{array}{l}\text { Clarke et al. } \\
\qquad(2002)^{(37)}\end{array}$ & $\begin{array}{l}\text { Cardiff \& Leeds/Bradford } \\
\quad \text { (England) } \\
\text { Postal sector }\end{array}$ & $\begin{array}{l}\text { Proximity to and } \\
\text { density of: } \\
\text { - Multiple store } \\
\text { - Co-operative store } \\
\text { - Gocery retail } \\
\text { - Discount store }\end{array}$ & $\begin{array}{l}\text { - Buffer (500 m around food } \\
\text { outlet) }\end{array}$ & $\begin{array}{l}\text { Cartairs index of deprivation } \\
\text { - Low/high household } \\
\text { - Retired/inactive } \\
\text { - No car indicator of Hansen } \\
\text { accessibility in Leeds/Bradford } \\
\text { and Cardiff }\end{array}$ & $\begin{array}{l}\text { The different indicators identified six } \\
\text { problematic food deserts: two in } \\
\text { Leeds/Bradford and four in Cardiff }\end{array}$ \\
\hline $\begin{array}{l}\text { Donkin et al. } \\
(1999)^{(38)}\end{array}$ & $\begin{array}{l}\text { London Town (England) } \\
\text { Postcode }\end{array}$ & $\begin{array}{l}\text { Distance to: } \\
\text { - Supermarket } \\
\text { - Greengrocer } \\
\text { - Butcher (halal or not) } \\
\text { - Other food outlets }\end{array}$ & $\begin{array}{l}\text { - Buffer (500 m around food } \\
\text { outlet) Euclidean distance } \\
\text { along the road }\end{array}$ & $\begin{array}{l}\text { - Cartairs deprivation scores } \\
\text { - Questionnaire of preferences } \\
\text { and perception of 'healthy diet' } \\
\text { for four major ethnic groups } \\
\text { - Price of seventy-one food items } \\
\text { - Density of population }\end{array}$ & $\begin{array}{l}\text { - Few areas where someone would } \\
\text { have to walk more than } 500 \mathrm{~m} \text { (along } \\
\text { the road) to reach to food outlet } \\
\text { - Relationship with population density: } \\
\text { distance to food outlet tended to be } \\
\text { lower in the area of highest population }\end{array}$ \\
\hline $\begin{array}{l}\text { Frank et al. } \\
\quad(2006)^{(47)}\end{array}$ & $\begin{array}{l}\text { Atlanta region (Georgia) } \\
\text { Elementary and middle } \\
\text { schools } \\
\text { Census tract }\end{array}$ & $\begin{array}{l}\text { Proximity to: } \\
\text { - Fast food } \\
\text { - Restaurant } \\
\text { - Convenience } \\
\text { - Grocery stores }\end{array}$ & $\begin{array}{l}\text { - Buffer }(0.25 \text { at } 1.25 \text { miles } \\
\text { around school) } \\
\text { - Road network distance }\end{array}$ & $\begin{array}{l}\text { Contextual data: } \\
\text { - Walkability } \\
\text { - Income } \\
\text { Spatial autocorrelation (Moran's I) } \\
\text { Detailed audit (food quality and } \\
\text { cost of foods for each type of } \\
\text { food outlets) }\end{array}$ & $\begin{array}{l}\text { - Spatial variation in type of food outlet } \\
\text { across neighbourhood by income, but } \\
\text { not by walkability }\end{array}$ \\
\hline $\begin{array}{l}\text { Jago et al. } \\
\quad(2007)^{(28)}\end{array}$ & $\begin{array}{l}\text { Houston (Texas) } \\
\text { Census tract } \\
204 \text { boy scouts } \\
\text { (10-14 years) }\end{array}$ & $\begin{array}{l}\text { Food stores and } \\
\text { restaurants: } \\
\text { - Proximity } \\
\text { - Density }\end{array}$ & $\begin{array}{l}\text { - Buffer (1 mile around home) } \\
\text { Number of food stores and } \\
\text { restaurants within each buffer } \\
\text { - Euclidean distance to the } \\
\text { nearest of each category of } \\
\text { food store and restaurant }\end{array}$ & $\begin{array}{l}\text { Individual variables } \\
\text { - Ethnicity, education, age } \\
\text { - BMI } \\
\text { - Consumption } \\
\text { (fruit, vegetables...) } \\
\text { Fruit and vegetable availability at } \\
\text { home }\end{array}$ & $\begin{array}{l}\text { Distance to the nearest small food store } \\
\text { but not proximity to large food store } \\
\text { was a positive predictor of fruit, juice, } \\
\text { low-fat and high-fat vegetable } \\
\text { consumption }\end{array}$ \\
\hline
\end{tabular}




\begin{tabular}{|c|c|c|c|c|c|}
\hline Author(s) (year) & $\begin{array}{l}\text { Location } \\
\text { Scale/population }\end{array}$ & $\begin{array}{l}\text { Measures food } \\
\text { availability }\end{array}$ & GIS method & Other variables & Findings \\
\hline $\begin{array}{l}\text { Jeffery et al. } \\
\qquad(2006)^{(29)}\end{array}$ & $\begin{array}{l}\text { Minnesota } \\
1033 \text { adults (18-76 years) }\end{array}$ & $\begin{array}{l}\text { Density of: } \\
\text { - Fast food } \\
\text { - Other restaurant }\end{array}$ & $\begin{array}{l}\text { - Buffer }(0 \cdot 5,1 \text { and } 2 \text { miles } \\
\text { around home and work } \\
\text { addresses) }\end{array}$ & $\begin{array}{l}\text { Individual variables } \\
\text { - Gender, education... } \\
\text { - BMI } \\
\text { - Hours of watched TV } \\
\text { - Physical activity } \\
\text { - Frequency eating at fast food }\end{array}$ & $\begin{array}{l}\text { - Positive association between 'eating } \\
\text { fast food' and having children, a high- } \\
\text { fat diet and BMl } \\
\text { - No association between measure of } \\
\text { fast food proximity and BMI }\end{array}$ \\
\hline $\begin{array}{l}\text { Laraia et al. } \\
\qquad(2004)^{(33)}\end{array}$ & $\begin{array}{l}\text { Wake County (North } \\
\text { Carolina) } \\
918 \text { pregnant women }\end{array}$ & $\begin{array}{l}\text { Distance to and } \\
\text { density of: } \\
\text { - Supermarket } \\
\text { - Convenience store } \\
\text { - Grocery store }\end{array}$ & $\begin{array}{l}\text { - Buffer ( } 0.5 \text { mile around home) } \\
\text { - Euclidean distance to the } \\
\text { closest food store }\end{array}$ & $\begin{array}{l}\text { - Diet quality index (FFQ) } \\
\text { Individual variables: age, } \\
\text { ethnicity, education income, } \\
\text { marital status }\end{array}$ & $\begin{array}{l}\text { Living at a distance greater than } 4 \text { miles } \\
\text { from a supermarket had a significant } \\
\text { negative association on the diet quality } \\
\text { of pregnant women }\end{array}$ \\
\hline $\begin{array}{l}\text { Larsen \& } \\
\text { Gilliliand } \\
(2008)^{(41)}\end{array}$ & $\begin{array}{l}\text { London (Ontario, Canada) } \\
\text { Census tract }\end{array}$ & $\begin{array}{l}\text { Supermarket: } \\
\text { - Density } \\
\text { Distance by: } \\
\text { - Public transit (bus) } \\
\text { - Street network }\end{array}$ & $\begin{array}{l}\text { - Service area }(500 \mathrm{~m} / 1 \mathrm{~km} \\
\text { around supermarket) } \\
\text { - Distance to the closest } \\
\text { supermarket } \\
\text { - Number of supermarkets } \\
\text { within } 1000 \mathrm{~m}\end{array}$ & $\begin{array}{l}\text { Composite index of socio- } \\
\text { economic distress } \\
\text { - Education } \\
\text { - Lone parenthood } \\
\text { - Unemployment } \\
\text { - Low income }\end{array}$ & $\begin{array}{l}\text { Food deserts exist particularly in the East } \\
\text { London area }\end{array}$ \\
\hline $\begin{array}{l}\text { Liu et al. } \\
\qquad(2007)^{(31)}\end{array}$ & $\begin{array}{l}\text { Marion County (Indiana) } \\
7334 \text { children ( } 3-18 \text { years) }\end{array}$ & $\begin{array}{l}\text { Distance to: } \\
\text { - Supermarket } \\
\text { - Grocery store } \\
\text { - Convenience store } \\
\text { - Fast food }\end{array}$ & $\begin{array}{l}\text { - Buffer ( } 2 \mathrm{~km} \text { around home) } \\
\text { - Network distance to the } \\
\text { closest food store }\end{array}$ & $\begin{array}{l}\text { Area level } \\
\text { - Population density } \\
\text { - Family income } \\
\text { Vegetation } \\
\text { - Satellite imagery } \\
\text { Individual } \\
\text { - BMI }\end{array}$ & $\begin{array}{l}\text { - Greener neigbourhoods are asso- } \\
\text { ciated with reduced risk of overweight } \\
\text { in children only in higher population } \\
\text { density neighbourhood } \\
\text { - Distance between children's home } \\
\text { and the closest supermarket was } \\
\text { associated with BMI in lower popula- } \\
\text { tion density neighbourhood }\end{array}$ \\
\hline $\begin{array}{l}\text { Moore et al. } \\
\qquad(2008 \mathrm{~b})^{(26)}\end{array}$ & $\begin{array}{l}\text { New York City } \\
\text { North Carolina } \\
\text { Maryland } \\
\text { Census tract } \\
2384 \text { adults (45-84 years) }\end{array}$ & $\begin{array}{l}\text { Supermarket: } \\
\text { - Density } \\
\text { Perceived availability of } \\
\text { healthy food }\end{array}$ & $\begin{array}{l}\text { - Kernel density method } \\
(1 \text { mile around home })\end{array}$ & $\begin{array}{l}\text { - Survey (MESA cohort) } \\
\text { - Index of dietary patterns and } \\
\text { eating behaviours (AHEI) } \\
\text { Respondents: } \\
\text { - Age, sex, race/ethnicity } \\
\text { - Househod income }\end{array}$ & $\begin{array}{l}\text { Population who had no supermarkets } \\
\text { close their homes less likely than } \\
\text { population in the highest category of } \\
\text { supermarket density to have a healthy } \\
\text { diet }\end{array}$ \\
\hline $\begin{array}{l}\text { Moore et al. } \\
\qquad(2008 \mathrm{a})^{(32)}\end{array}$ & $\begin{array}{l}\text { New York city } \\
\text { North Carolina } \\
\text { Maryland } \\
\text { Census tract } \\
5774 \text { adults (45-84 years) }\end{array}$ & $\begin{array}{l}\text { Densities of: } \\
\text { - Supermarkets } \\
\text { - Other smaller stores } \\
\text { Perceived availability of } \\
\quad \text { healthy food }\end{array}$ & $\begin{array}{l}\text { - Kernel density method } \\
(1 \text { mile around home })\end{array}$ & $\begin{array}{l}\text { Respondents (MESA cohort) } \\
\text { - Race/ethnicity } \\
\text { - Income } \\
\text { Census tract: } \\
\text { - Population density }\end{array}$ & $\begin{array}{l}\text { Relationship between supermarket } \\
\text { density and perceived availability of } \\
\text { healthy foods: residents who lived in } \\
\text { areas with the lowest densities of } \\
\text { supermarket rated availability of } \\
\text { healthy foods lower than those in } \\
\text { areas with the highest densities of } \\
\text { supermarkets }\end{array}$ \\
\hline
\end{tabular}




\begin{tabular}{|c|c|c|c|c|c|}
\hline Author(s) (year) & $\begin{array}{l}\text { Location } \\
\text { Scale/population }\end{array}$ & $\begin{array}{l}\text { Measures food } \\
\text { availability }\end{array}$ & GIS method & Other variables & Findings \\
\hline $\begin{array}{l}\text { O'Dwyer \& } \\
\text { Coveney } \\
\left(_{(2006)^{(35)}}\right.\end{array}$ & $\begin{array}{l}\text { Adelaide (Australia) } \\
\text { LGA }\end{array}$ & $\begin{array}{l}\text { Supermarket: } \\
\text { - Density } \\
\text { - Proximity }\end{array}$ & $\begin{array}{l}\text { - Buffer ( } 2.5 \mathrm{~km} \text { around area } \\
\text { (LGA)) } \\
\text { - Distance by road network to } \\
\text { the closest supermarket }\end{array}$ & SEIFA & $\begin{array}{l}\text { Socio-economic differences in access to } \\
\text { food and the availability of food outlets } \\
\text { Food deserts appear to exist }\end{array}$ \\
\hline $\begin{array}{l}\text { Pearce et al. } \\
\quad(2006)^{(43)}\end{array}$ & $\begin{array}{l}\text { New Zealand } \\
\text { Census meshblock (smallest } \\
\text { unit of dissemination of } \\
\text { census data) }\end{array}$ & $\begin{array}{l}\text { Travel time for: } \\
\text { - Recreational } \\
\text { amenities } \\
\text { - Food outlet } \\
\text { - Educational facilities } \\
\text { - Health facilities }\end{array}$ & $\begin{array}{l}\text { Modelling travel time depending on: } \\
\text { - Speed limits } \\
\text { - Type of road surface } \\
\text { - Sinuosity/topograpy }\end{array}$ & & $\begin{array}{l}\text { Strong geographical variations in } \\
\text { community resource accessibility } \\
\text { between neighbourhoods within an } \\
\text { urban area }\end{array}$ \\
\hline $\begin{array}{l}\text { Pearce et al. } \\
\quad(2007)^{(42)}\end{array}$ & $\begin{array}{l}\text { New Zealand } \\
\text { School } \\
\text { Census meshblock }\end{array}$ & $\begin{array}{l}\text { Travel time and } \\
\text { distance to: } \\
\text { - Fast food } \\
\text { - Supermarkets } \\
\text { - Convenience store } \\
\text { for each school }\end{array}$ & $\begin{array}{l}\text { Modelling travel time to each } \\
\text { - School } \\
\text { - Census meshblock }\end{array}$ & $\begin{array}{l}\text { - NZDep - } 2001 \\
\text { - Socio-economic characteristics } \\
\text { of school } \\
\text { - Urban/rural status }\end{array}$ & $\begin{array}{l}\text { Access to fast food outlets is better in } \\
\text { more deprived neighbourhoods and } \\
\text { around more socio-economically } \\
\text { disadvantaged schools }\end{array}$ \\
\hline $\begin{array}{l}\text { Pearce et al. } \\
(2008)^{(23)}\end{array}$ & $\begin{array}{l}\text { New Zealand } \\
\text { Census meshblock } \\
12529 \text { adults (15 years } \\
\text { and more) }\end{array}$ & $\begin{array}{l}\text { Travel time to: } \\
\text { - Supermarket } \\
\text { - Convenience store }\end{array}$ & $\begin{array}{l}\text { Modelling travel time } \\
\text { depending on: } \\
\text { - Speed limits } \\
\text { - Type of road surface } \\
\text { - Sinuosity/topograpy }\end{array}$ & $\begin{array}{l}\text { - Consumption of the } \\
\text { recommended daily intake } \\
\text { of fruit and vegetable } \\
\text { - Individual variables } \\
\text { - Meshblock variables: } \\
\text { NZDep - } 2001\end{array}$ & $\begin{array}{l}\text { Little evidence that poor locational } \\
\text { access to food retail provision is } \\
\text { associated with lower fruit and } \\
\text { vegetable consumption }\end{array}$ \\
\hline $\begin{array}{l}\text { Sharkey \& Horel } \\
(2008)^{(51)}\end{array}$ & $\begin{array}{l}\text { Six counties rural region } \\
\text { of Texas } \\
\text { Census block group }\end{array}$ & $\begin{array}{l}\text { Distance to: } \\
\text { - Supermarket } \\
\text { - Grocery store } \\
\text { - Convenience store } \\
\text { - Discount store }\end{array}$ & $\begin{array}{l}\text { Distance by road to the closest } \\
\text { food store }\end{array}$ & $\begin{array}{l}\text { - Deprivation Index } \\
\text { - Minority composition } \\
\text { - Population density }\end{array}$ & $\begin{array}{l}\text { Better spatial access to food store for } \\
\text { neighbourhood with high socio- } \\
\text { economic deprivation }\end{array}$ \\
\hline $\begin{array}{l}\text { Smoyer-Tomic } \\
\text { et al. } \\
(2008)^{(40)}\end{array}$ & $\begin{array}{l}\text { City of Edmonton (Alberta, } \\
\text { Canada) } \\
\text { Census block }\end{array}$ & $\begin{array}{l}\text { Proximity to: } \\
\text { - Supermarket } \\
\text { - Fast food }\end{array}$ & $\begin{array}{l}\text { - Buffer }(500,800 \mathrm{~m} \text { around } \\
\text { geometric centre of each } \\
\text { census block) } \\
\text { - Distance to the nearest outlet } \\
\text { by street network }\end{array}$ & $\begin{array}{l}\text { Area level } \\
\text { - Race/ethnicity, SES } \\
\text { - Age, family status } \\
\text { - Housing tenure, urbanisation }\end{array}$ & $\begin{array}{l}\text { Fast food outlet exposure was higher in } \\
\text { low-income neighbourhood }\end{array}$ \\
\hline $\begin{array}{l}\text { Timperio et al. } \\
\quad(2008)^{(24)}\end{array}$ & $\begin{array}{l}\text { Greater Melbourne } \\
\text { (Geelong area) (Australia) } \\
801 \text { children (5-6/10-12 } \\
\text { years) }\end{array}$ & $\begin{array}{l}\text { Distance to: } \\
\text { - Supermarket } \\
\text { - Greengrocer } \\
\text { - Convenience store } \\
\text { - Fast food, take-away } \\
\text { - Restaurant, cafés to } \\
\text { the closest outlet }\end{array}$ & $\begin{array}{l}\text { - Buffer ( } 800 \mathrm{~m} \text { around home) } \\
\text { - Existence of one or more outlet } \\
\text { - Distance by road }\end{array}$ & $\begin{array}{l}\text { - Children: frequency of con- } \\
\text { sumption of fruit or vegetables } \\
\text { - Parents: sociodemographic } \\
\text { variables }\end{array}$ & $\begin{array}{l}\text { The availability of convenience stores } \\
\text { and fast food outlets close to home } \\
\text { may have a detrimental effect on } \\
\text { children's fruit and vegetable intake } \\
\text { - The likelihood to consuming vegetables } \\
\text { was greater the further children lived } \\
\text { from a supermarket or a fast food outlet }\end{array}$ \\
\hline
\end{tabular}


Table 1 Continued

\begin{tabular}{|c|c|c|c|c|c|}
\hline Author(s) (year) & $\begin{array}{l}\text { Location } \\
\text { Scale/population }\end{array}$ & $\begin{array}{l}\text { Measures food } \\
\text { availability }\end{array}$ & GIS method & Other variables & Findings \\
\hline $\begin{array}{l}\text { Wang et al. } \\
(2007)^{(30)}\end{array}$ & $\begin{array}{l}\text { Agricultural regions of } \\
\text { California } \\
\text { Census tract block } \\
7595 \text { adults (25-74 years) }\end{array}$ & $\begin{array}{l}\text { For different type of } \\
\text { retail food stores and } \\
\text { fast foods: } \\
\text { - Density } \\
\text { - Proximity }\end{array}$ & $\begin{array}{l}\text { - Buffer ( } 0.5 \text { mile around home) } \\
\text { - Shortest Euclidean distance }\end{array}$ & $\begin{array}{l}\text { - Index of the neighbourhood } \\
\text { socio-economic environment } \\
\text { - Individual-level variables } \\
\text { (sex, age, smoking, } \\
\text { physical activity...) } \\
\text { - BMI }\end{array}$ & $\begin{array}{l}\text { - Proximity to various type of food } \\
\text { stores was associated with neigh- } \\
\text { bourhood SES } \\
\text { - Higher BMI among residents of low } \\
\text { socio-economic neighbourhoods } \\
\text { - Living in an environment where } \\
\text { healthy food is not readily available is } \\
\text { associated with increased obesity risk }\end{array}$ \\
\hline $\begin{array}{l}\text { Winkler } \\
\quad(2006)^{(36)}\end{array}$ & $\begin{array}{l}\text { Brisbane (Australia) } \\
\text { Census collection districts }\end{array}$ & $\begin{array}{l}\text { Distance to the nearest } \\
\text { types of shops selling } \\
\text { fruits and vegetables } \\
\text { (supermarket, } \\
\text { greengrocers...) }\end{array}$ & $\begin{array}{l}\text { - Buffer ( } 2.5 \mathrm{~km} \text { around area) } \\
\text { - Shortest Euclidean distance }\end{array}$ & $\begin{array}{l}\text { - IRSD } \\
\text { - The opening hours of nearby } \\
\text { fruit and vegetable outlets }\end{array}$ & $\begin{array}{l}\text { It is unlikely that living in a socio- } \\
\text { economically disadvantged area } \\
\text { presents fewer opportunities to } \\
\text { purchase fruits and vegetables, at } \\
\text { least in an urban area }\end{array}$ \\
\hline $\begin{array}{l}\text { Zenk et al. } \\
\quad(2005)^{(63)}\end{array}$ & $\begin{array}{l}\text { Detroit (Michigan) } \\
\text { Census tract block }\end{array}$ & $\begin{array}{l}\text { Supermarket: } \\
\text { - Proximity }\end{array}$ & $\begin{array}{l}\text { - Manhattan distance distance to } \\
\text { the closest supermarket }\end{array}$ & $\begin{array}{l}\text { - Population density } \\
\text { - Non-Hispanic African } \\
\text { American residents } \\
\text { - Residents below the } \\
\text { poverty line } \\
\text { - Spatial autocorrelation } \\
\text { (Moran's I) }\end{array}$ & $\begin{array}{l}\text { The most impoverished neighbourhoods } \\
\text { in which African Americans resided } \\
\text { were further from the nearest } \\
\text { supermarket than were the most } \\
\text { impoverished white neighbourhoods }\end{array}$ \\
\hline $\begin{array}{l}\text { Zenk \& Powell } \\
\qquad(2008)^{(46)}\end{array}$ & $\begin{array}{l}\text { Fifty states and twenty } \\
\text { largest cities in USA } \\
\text { School } \\
\text { Census tract }\end{array}$ & $\begin{array}{l}\text { Density to: } \\
\text { - Fast food } \\
\text { - Convenience store }\end{array}$ & - Buffer ( 0.5 mile around school) & $\begin{array}{l}\text { Independant data } \\
\text { - Racial/ethnic } \\
\text { - Median income } \\
\text { - School level } \\
\text { - Population density } \\
\text { - Level of urbanisation } \\
\text { - Student body size }\end{array}$ & $\begin{array}{l}\text { Within } 0.5 \text { miles (walking distance): } \\
\text { - Fast food and convenience stores are } \\
\text { more available in lowest-income } \\
\text { neighbourhood (except in African } \\
\text { American neighbourhood) } \\
\text { - Afican American neighbourhoods } \\
\text { have fewer food outlets than white } \\
\text { neighbourhoods } \\
\text { - Urban high schools are exposed to } \\
61 \% \text { more fast food than urban middle } \\
\text { schools }\end{array}$ \\
\hline
\end{tabular}

GIS, geographic information systems; LGA, local government area; SES, socio-economic status; SEIFA, Socio-Economic Index For Areas; TV, television; MESA, Multi-Ethnic Study of Atherosclerosis; AHEI, Alternate Healthy Eating Index; NZDep, New Zealand deprivation index; IRSD; Index of Relative Socio-economic Disadvantage. 
involved census tracts and postal sectors in North American studies, wards and postal codes in the United Kingdom and census meshblocks in Australia and New Zealand (Table 1). Most studies were based on census tracts, since they had been conducted in the United States (seventeen out of twenty-nine studies), while four were performed in Australia $^{(24,34-36)}$, two in the United Kingdom ${ }^{(37,38)}$, three in Canadian cities ${ }^{(39-41)}$ and three in New Zealand ${ }^{(23,42,43)}$.

Two studies were related to fast food outlets only ${ }^{(44,45)}$, one to fast food and convenience stores ${ }^{(46)}$ and one to fast food, full-service restaurants, convenience and grocery stores ${ }^{(47)}$. The remaining studies focused on a common type of food store: the supermarket. In all of these studies, residential contexts were characterised by socio-economic indicators (including unemployment rates and single-parent rates ${ }^{(39,41)}$, income $^{(31)}$, race/ethnicity ${ }^{(46,48)}$, households without cars ${ }^{(37,49)}$ ) and by other information such as degree of commercialisation $^{(44)}$, urban/rural status ${ }^{(42,46)}$, safety ${ }^{(27)}$ and neighbourhood walkability $^{(47)}$ (environmental attributes that encourage walking ${ }^{(50)}$ ). In nine out of eighteen studies, an index of deprivation (constructed from census data) was used to describe the social-residential context ${ }^{(23,34,35,37-39,41,42,51)}$.

\section{GIS measurements of the food environment}

In the articles reviewed, two main notions were used to assess the food environment: density and proximity. (i) Density is usually the number of food outlets (food stores, restaurants) in an administratively defined area (census or postal units) or an area defined by the authors (specific zone). (ii) Proximity is defined between two locations such as respondent address (home, school) and the closest food outlet. It could be measured by a straight-line distance (Euclidean distance) or by travel time (time needed to travel to a food outlet). Table 2 lists the various methods described in the literature concerning the food environment used for assessing density and proximity, along with the number of corresponding studies for each method. Among the twentynine studies examined, twelve combined both spatial approaches (Table 2).

\section{Density}

Buffer

The most common GIS approach (eighteen studies out of twenty-nine) was the buffer. This consists of defining a zone around a given location within a specified distance (or shape). The location can be a point (home, school, work or food outlet address), a line (road) or a polygon (neighbourhood).

Most studies defined buffers in order to quantify the availability or accessibility of food outlets. Seven of these studies used a buffer zone around the respondent's home $e^{(22,24,25,28-31)}$, three around the school $^{(44,46,47)}$, four around the food store ${ }^{(37,38,41,49)}$ and four around the centroid (geometric center) of each neighbourhood ${ }^{(35,36,40,45)}$. For one of these studies, analyses were performed using
Table 2 Summary measures of food availability used in twentynine published articles

\begin{tabular}{lc}
\hline GIS measure of food accessibility & Number of studies \\
\hline Density & \\
Buffer & 18 \\
Circular & 11 \\
Network & 7 \\
Kernel density & 2 \\
Spatial clustering & 1 \\
Proximity & \\
Euclidean distance & 7 \\
Manhattan distance & 1 \\
Distance by road or street & 8 \\
Modelling travel time & 4
\end{tabular}

GIS, geographic information systems.

Note: Total number of studies was more than twenty-nine because twelve studies combined measurements of density and proximity.

buffers around both the home and the work address ${ }^{(29)}$, while only one study combined a buffer around a point (supermarket) or around a line (bus route) ${ }^{(41)}$. It should be noted that there are two ways to define the shape of a buffer for the GIS user. It can be constructed either by a zone surrounding a location (circular buffer when the given location is a point) or by a zone along the street network (network buffer; e.g. see figures in Frank et al. ${ }^{(52)}$ ).

\section{Circular buffer}

In the studies we reviewed, the values used for the radius of a circular buffer were between 100 and $2500 \mathrm{~m}$. Depending on the study, these distances were selected on the basis of estimations of neighbourhood walkability or distances that individuals might be ready to cover to reach food outlets $^{(24,28,44,45)}$. In a study by Bodor et $a l^{(22)}$, different distances were chosen according to the type of food store: $100 \mathrm{~m}$ for small food stores (e.g. the approximate size of a city block) and $1000 \mathrm{~m}$ for large supermarkets. Two authors $^{(35,36)}$ used a much wider radius of $2500 \mathrm{~m}$ around the geometric centre of the neighbourhood to define the area in which residents were likely to shop.

\section{Network buffer}

A network buffer can be defined as being based on the accessibility of food outlets via the mode of transportation used and the type of destination. Larsen and Gilliland ${ }^{(41)}$ used two network buffers in the town of London (Ontario, Canada). The first buffer was based on a distance of $1000 \mathrm{~m}$ by foot around each supermarket. The second buffer was created around each bus route to estimate a $500 \mathrm{~m}$ network service line area with public transport access to supermarkets.

\section{Kernel density estimation}

Kernel density is a spatial smoothing method employed to transform a sample of geographically referenced point data (e.g. address of food outlet) into a smooth continuous surface $^{(53-56)}$. As described by Kloog et al. ${ }^{(57)}$, the general principles of this statistical technique are to estimate the 
'intensity of referenced points across a surface, by calculating the overall number of cases situated within a given search radius from a target point'. A distance function is introduced in the calculation so that 'points lying near the centre of the search area are weighted more heavily than those lying near the edge ${ }^{(57)}$. The various steps for generating kernel densities with GIS software have been described by Guagliardo ${ }^{(58)}$.

Only two studies, both by Moore et al. ${ }^{(26,32)}$, used kernel density estimation to assess the spatial distribution of food outlets (Table 2). In that case, the aim was to create a smooth map of food store density per square mile where the home location proximity was emphasised and more weight was put on closer outlets.

\section{Spatial clustering}

A spatial scan statistic is used to assess whether events are randomly distributed within the study area, and if not, to identify significant spatial clusters ${ }^{(59,60)}$. This method consists of creating moving windows of various shapes (circles, squares) and sizes (radius, sides of square). These windows are moved systematically across the map, which enables assessment of the likelihood that events are more prevalent inside than outside that given window (see SatScan process $\left.^{(61)}\right)$. With this method, Baker et al. ${ }^{(48)}$ identified spatial neighbourhood variation in the rate of supermarkets and fast food outlets in St Louis, MO, United States, and observed clusters of food supermarkets and fast food outlets (i.e. areas with higher or lower rates than expected).

\section{Network analysis and proximity measures}

\section{Proximity defined as a distance}

Several types of distances are typically used to assess proximity with GIS: Euclidean distance (straight line distance), Manhattan (city block distance) and network distance. The Manhattan distance corresponds to the distance between two points measured along axes at right angles ${ }^{(62)}$. In other words, Manhattan distance represents an approximate distance close to a street map and is mainly used on squared city maps.

In our review, six studies measured the distance between home/school and food outlets via the Euclidean distance ${ }^{(22,28,30,33,39,44)}$ (Table 2). In Eastside Detroit areas with no supermarket, Zenk et al. ${ }^{(63)}$ used the Manhattan distance to evaluate the shortest distance between home addresses and food outlets in a population of AfricanAmerican women. Two studies used network distance by $\operatorname{road}^{(24,63)}$. In other studies, the network distance by street travel was used to evaluate the minimum distance residents must walk from their home/school to the closest food outlet ${ }^{(27,31,38,40,41,47)}$.

\section{Proximity measured by travel time}

The travel time between a given place (e.g. school or home address) and the address of a food outlet can be calculated by GIS according to the means of transport and the specificities of the network. Four out of twenty-nine studies used travel time as a proximity measurement ${\text { (Table } 1)^{(23,34,42,43)} \text {. Burns and Inglis }}^{(34)}$ developed a travel time model between home, fast food outlet and supermarket according to a number of variables including means of transport (car, bus, on foot), type of road (speed limit), topography (barriers as rivers or railway lines) and other characteristics of the public transport network (i.e. frequency of buses). Travel time for each type of transport was compared between underprivileged and privileged neighbourhoods, with the latter having better access to supermarkets.

\section{Discussion}

In this review, we investigated which GIS methods have been used to define the food environment and the types of spatial measurements they generate. We found twenty-nine articles that reported GIS methods for measuring spatial accessibility of food outlets as a key feature of the local food environment. We identified two main types of spatial measures to quantify the food environment: density and proximity. The density approach quantifies the availability of food outlets using the buffer method, kernel density estimation or spatial clustering. The proximity approach assesses the distance to food outlets by measurements of distance or travel times. Numerous studies combined both approaches.

\section{How do GIS methods contribute to research on the food environment?}

It is clear from the present work that the number of studies that include geographic measurements of density and/or proximity of food outlets as operational variables in the food environment have increased rapidly in recent years. Twenty-two of the twenty-nine articles examined here were published between January 2006 and June 2008. It is likely that the continuous refinement of GIS software and the increased availability of precisely geocoded databases have contributed substantially, and will continue to contribute, to this increase ${ }^{(19)}$.

In the studies included in this review, two approaches based on GIS methods were used to characterise the local food environment. One involved assessing the number of food outlets in an area (density) and the other assessed proximity to facilities. Interestingly, a large number of studies combined both approaches. Indeed, as argued by Apparicio et $a l^{(39)}$, a single measure of access cannot fully describe accessibility of food outlets. Focusing on the issue of 'food desert' (areas characterised by relatively poor access to healthy and affordable food ${ }^{(64)}$ ), Apparicio et $a l^{(39)}$ proposed a methodology based on three measurements of access using the shortest network distance: diversity, proximity and variety (average distance to the three closest different chain-name supermarkets). 
An important advantage of the GIS approach is that it enables assessment of spatial variations in prevalence independently of administrative boundaries ${ }^{(65)}$. Many phenomena are continuously distributed over space and are independent of arbitrarily defined boundaries ${ }^{(66,67)}$. Estimating the density of food outlets within buffers, or by means of kernel density estimation rather than administrative area, enables one to take into account the fact that individuals often cross the boundaries of their residential area to go shopping. However, it should be emphasised that the appropriate size of the area around the place of residence to be defined as the neighbourhood remains subject to debate ${ }^{(68-70)}$. The choice of this area size is based on assumptions concerning the geographic zone that includes food environment elements influencing food behaviour. In the studies reviewed here, the distance used to define the residential area varied depending on different criteria such as the age of the respondent, type of food outlet and type of transportation. It is also important to underline that few studies exist which question individuals as to the distance they would be prepared to cover for food needs. Thus, because of the complexity of the relationship between environment and behaviour, defining the size of the neighbourhood in which this relationship operates remains a challenging methodological issue ${ }^{(68,71)}$.

GIS methods enable the modelling of proximity to food outlets using metric distance and travel time to food outlets. In general, modelling of travel time using the GIS leads to more realistic measurements (taking into account speed limit, topography and network complexity) than the usual mathematical distances, particularly at the local level in sub-metropolitan areas ${ }^{(62)}$ or in rural areas ${ }^{(72)}$. However, the use of this travel time model, which requires spatial information, is more complex than calculating the mathematical distances between two points.

In the articles that we reviewed, which used travel time to food outlets, the car was the type of transportation evaluated in four papers, with public transport evaluated in only one ${ }^{(34)}$. None dealt with travel time by foot or 'mixed' travel. This is an important point because families with low income may not own a car or even have access to public transportation. In future studies, a methodological challenge therefore lies in measuring travel time from the respondent's address to food outlets according to the different types of transport available (car, public transport, or on foot). In addition, modelling travel time according to public transport or on foot requires more sophisticated GIS modelling than private car transport ${ }^{(73)}$.

On the other hand, Larsen et al. ${ }^{(41)}$ showed that, with the GIS, the geographic distribution of supermarkets has changed over time, thus influencing the relationship between people and places in a spatial access approach. Through GIS use, it is possible to capture the temporal changes in localisation of food outlets and land use, which will improve our understanding of the relationship between food environment and food behaviour over time ${ }^{(19,74)}$.

One of the major challenges when using GIS for studying the food environment concerns the quality of the data available. The validity of GIS-based measures of environmental features of the food environment has recently been discussed $^{(19)}$. Since street addresses of facilities were often obtained from commercial databases or had been collected for other purposes, data accuracy and comprehensiveness must be viewed with caution ${ }^{(71,75)}$. In addition, there may exist a mismatch between the geocoded location of a facility and its true location, e.g. via the GPS (global positioning system) technique ${ }^{(75,76)}$.

\section{A major challenge: which concepts should be used to characterise access?}

The articles reviewed here focused on spatial access as estimated by GIS methods. Nevertheless, it should be noted that few authors specifically use the term 'spatial' or 'geographic' when dealing with the broad concept of access $^{(39,51)}$. Access that includes material and social dimensions is a complex notion, and geographic proximity does not systematically imply accessibility. Gould ${ }^{(77)}$ describes accessibility as 'a notion difficult to grasp... one of these common terms everybody uses until the problem arises of defining and measuring the concept'. Penchansky and Thomas ${ }^{(78)}$ defined five dimensions for access, including availability, accessibility, affordability, acceptability and accommodation. Only the first two dimensions, corresponding to spatial measures, reflect the geographic distribution (e.g. of facilities around the home address) and can be estimated by GIS methods. This may be viewed as a possible weakness of these methods. However, by definition, the other dimensions reflecting the cultural, social and economic factors are not taken into account.

The 'ideal' study of access to food outlets would appear to be one that associates all dimensions related to accessibility: proximity, diversity, availability, affordability (cost) and perception, with the term 'diversity' referring to the types of food outlets and 'availability' referring to the food supply at the food outlets. Only four of the articles ${ }^{(22,47-49)}$ combined assessment of spatial access to food outlets with an evaluation of the actual food content of the outlet. Among those articles, only two took into account cost and quality ${ }^{(47,49)}$ in addition to the availability of foods, especially healthy foods. Access to food outlets may also be limited by the subject's perception of the environment in his/her neigbourhood ${ }^{(32,79)}$. Moore et $a l^{(32)}$ suggested that the availability of healthy foods as reported by residents (perception) and their availability as measured by GIS application (density) provide complementary information for characterising the local food environment. In other words, methodology for conducting an 'ideal' research study would have to combine GIS potential and survey approaches to describe both spatial and social accessibility of healthy foods. 


\section{Conclusions}

Accessibility to services and facilities and, in particular, to healthy food, is an important social equity issue ${ }^{(39)}$. Geographic analysis models may provide local authorities and policy makers with new views and possibilities for making decisions as to the location of services in order to offer a fair choice to the entire population. For example, Banos et al. ${ }^{(54,80)}$ have designed a GIS application that identifies hot spots by spatial regression ${ }^{(81)}$. These results enabled the targeting of parts of the road network that needed modifications ${ }^{(80)}$. Gatrell and Naumann ${ }^{(82)}$ adapted this tool to the field of health-care and suggested potential sites for building new hospitals, with various scenarios being examined according to traffic density.

It should also be noted that spatial accessibility of healthy food is only one of the multiple determinants of a healthy lifestyle, as emphasised by socio-ecological models of behaviours ${ }^{(2-4)}$. Further development of spatial analysis methods should help to better define its importance in various settings ${ }^{(83)}$. On the basis of the articles reviewed here, we suggest two avenues for future methodological research when analysing accessibility of facilities relevant to food behaviour. First, there is a need to test and compare more sophisticated spatial GIS modelling such as travel time or potential model principles and gravity models ${ }^{(58,84)}$. The latter combine diversity (type of facilities) and accessibility by using distribution of facilities throughout the area, together with a distance function to calculate the attractiveness of a food outlet (catchment area). Second, future research should benefit from a combination of GIS methods and survey approaches to describe both spatial and social food outlet accessibility, and to better understand how the food environment influences food behaviour and health.

\section{Acknowledgements}

This work is part of the ELIANE (Environmental LInks to physical Activity, Nutrition and hEalth) study. ELIANE is a project supported by the French National Research Agency (Agence Nationale de la Recherche, ANR-07PNRA-004). The authors declare that they have no competing interests. H.C. designed the study, performed the literature search and data extraction, and drafted the manuscript. J.-M.O. supervised the study design and data collection, and contributed to the finalisation of the paper. R.C., P.S., C.S., B.C., A.B., D.B. and C.W. assisted with the literature search and the writing of the manuscript. J.-M.O. is the coordinator of the ELIANE study; C.S., B.C. and C.W. are the principal investigators in the ELIANE study. All the authors read and approved the final version of the manuscript.

\section{References}

1. Story M, Kaphingst KM, Robinson-O'Brien R et al. (2008) Creating healthy food and eating environments: policy and environmental approaches. Annu Rev Public Health 29, 253-272.

2. Sallis JF \& Glanz K (2009) Physical activity and food environments: solutions to the obesity epidemic. Milbank Q 87, 123-154.

3. Booth KM, Pinkston MM \& Poston WS (2005) Obesity and the built environment. J Am Diet Assoc 105, S110-S117.

4. Townshend T \& Lake AA (2009) Obesogenic urban form: theory, policy and practice. Health Place 15, 909-916.

5. Kawachi I \& Berkman LF (2003) Neighborbood and Health. New York: Oxford University Press.

6. Glanz K, Sallis JF, Saelens BE et al. (2005) Healthy nutrition environments: concepts and measures. Am J Health Promot 19, 330-333, ii.

7. Glanz K (2009) Measuring food environments: a historical perspective. Am J Prev Med 36, S93-S98.

8. Papas MA, Alberg AJ, Ewing R et al. (2007) The built environment and obesity. Epidemiol Rev 29, 129-143.

9. Hill JO \& Peters JC (1998) Environmental contributions to the obesity epidemic. Science 280, 1371-1374.

10. Larson N, Story M \& Nelson M (2009) Neighborhood environments, disparities in access to healthy foods in the US. Am J Prev Med 36, 74-81.

11. Holsten JE (2008) Obesity and the community food environment: a systematic review. Public Health Nutr 12, 1-9.

12. White M (2007) Food access and obesity. Obes Rev $\mathbf{8}$, Suppl. 1, 99-107.

13. Rose D \& Richards R (2004) Food store access and household fruit and vegetable use among participants in the US Food Stamp Program. Public Health Nutr 7, 1081-1088.

14. Inglis V, Ball K \& Crawford D (2008) Socioeconomic variations in women's diets: what is the role of perceptions of the local food environment? J Epidemiol Community Health 62, 191-197.

15. McKinnon RA, Reedy J, Morrissette MA et al. (2009) Measures of the food environment: a compilation of the literature, 1990-2007. Am J Prev Med 36, S124-S133.

16. Longley PA, Goodchild MF, Maguire D et al. (2005) Geographic Information Systems and Science. Chichester: John Wiley \& Sons.

17. McLafferty SL (2003) GIS and health care. Annu Rev Public Health 24, 25-42.

18. Wendel-Vos W, Droomers M, Kremers S et al. (2007) Potential environmental determinants of physical activity in adults: a systematic review. Obes Rev 8, 425-440.

19. Matthews SA, Moudon AV \& Daniel M (2009) Work group II: using geographic information systems for enhancing research relevant to policy on diet, physical activity, and weight. Am J Prev Med 36, S171-S176.

20. McKinnon RA, Reedy J, Handy SL et al. (2009) Measuring the food and physical activity environments: shaping the research agenda. Am J Prev Med 36, S81-S85.

21. Liese AD, Weis KE, Pluto D et al. (2007) Food store types, availability, and cost of foods in a rural environment. $J \mathrm{Am}$ Diet Assoc 107, 1916-1923.

22. Bodor JN, Rose D, Farley TA et al. (2008) Neighbourhood fruit and vegetable availability and consumption: the role of small food stores in an urban environment. Public Health Nutr 11, 413-420.

23. Pearce J, Hiscock R, Blakely T et al. (2008) The contextual effects of neighbourhood access to supermarkets and convenience stores on individual fruit and vegetable consumption. J Epidemiol Community Health 62, 198-201. 
24. Timperio A, Ball K, Roberts R et al. (2008) Children's fruit and vegetable intake: associations with the neighbourhood food environment. Prev Med 46, 331-335.

25. Laraia BA, Messer L, Kaufman JS et al. (2006) Direct observation of neighborhood attributes in an urban area of the US south: characterizing the social context of pregnancy. Int J Health Geogr 5, 11.

26. Moore LV, Diez Roux AV, Nettleton JA et al. (2008) Associations of the local food environment with diet quality - a comparison of assessments based on surveys and geographic information systems: the multi-ethnic study of atherosclerosis. Am J Epidemiol 167, 917-924.

27. Burdette HL \& Whitaker RC (2004) Neighborhood playgrounds, fast food restaurants, and crime: relationships to overweight in low-income preschool children. Prev Med 38, 57-63.

28. Jago R, Baranowski T, Baranowski JC et al. (2007) Distance to food stores \& adolescent male fruit and vegetable consumption: mediation effects. Int J Behav Nutr Phys Act 4, 35 .

29. Jeffery RW, Baxter J, McGuire M et al. (2006) Are fast food restaurants an environmental risk factor for obesity? Int $J$ Behav Nutr Phys Act 3, 2.

30. Wang MC, Kim S, Gonzalez AA et al. (2007) Socioeconomic and food-related physical characteristics of the neighbourhood environment are associated with body mass index. J Epidemiol Community Health 61, 491-498.

31. Liu GC, Wilson JS, Qi R et al. (2007) Green neighborhoods, food retail and childhood overweight: differences by population density. Am J Health Promot 21, 317-325.

32. Moore LV, Diez Roux AV \& Brines S (2008) Comparing perception-based and Geographic Information System (GIS)-based characterizations of the local food environment. J Urban Health 85, 206-216.

33. Laraia BA, Siega-Riz AM, Kaufman JS et al. (2004) Proximity of supermarkets is positively associated with diet quality index for pregnancy. Prev Med 39, 869-875.

34. Burns CM \& Inglis AD (2007) Measuring food access in Melbourne: access to healthy and fast foods by car, bus and foot in an urban municipality in Melbourne. Health Place 13, 877-885.

35. O'Dwyer LA \& Coveney J (2006) Scoping supermarket availability and accessibility by socio-economic status in Adelaide. Health Promot J Aust 17, 240-246.

36. Winkler E, Turrell G \& Patterson C (2006) Does living in a disadvantaged area mean fewer opportunities to purchase fresh fruit and vegetables in the area? Findings from the Brisbane food study. Health Place 12, 306-319.

37. Clarke G, Eyre H \& Guy C (2002) Deriving indicators of access to food retail provision in British cities: studies of Cardiff, Leeds and Bradford. Urban Stud 30, 2041-2060.

38. Donkin A, Dowler E, Stevenson S et al. (1999) Mapping access to food at a local level. Br Food J 101, 554-564.

39. Apparicio P, Cloutier MS \& Shearmur R (2007) The case of Montreal's missing food deserts: evaluation of accessibility to food supermarkets. Int J Health Geogr 6, 4.

40. Smoyer-Tomic KE, Spence JC, Raine KD et al. (2008) The association between neighborhood socioeconomic status and exposure to supermarkets and fast food outlets. Health Place 14, 740-754.

41. Larsen K \& Gilliland J (2008) Mapping the evolution of 'food deserts' in a Canadian city: supermarket accessibility in London, Ontario, 1961-2005. Int J Health Geogr 7, 16.

42. Pearce J, Blakely T, Witten K et al. (2007) Neighborhood deprivation and access to fast-food retailing: a national study. Am J Prev Med 32, 375-382.

43. Pearce J, Witten K \& Bartie P (2006) Neigbourhoods and health: a GIS approach to measuring community resource accessibility. J Epidemiol Community Health 60, 389-395.
44. Austin SB, Melly SJ, Sanchez BN et al. (2005) Clustering of fast-food restaurants around schools: a novel application of spatial statistics to the study of food environments. Am J Public Health 95, 1575-1581.

45. Block JP, Scribner RA \& DeSalvo KB (2004) Fast food, race/ ethnicity, and income: a geographic analysis. Am J Prev Med 27, 211-217.

46. Zenk SN \& Powell LM (2008) US secondary schools and food outlets. Health Place 14, 336-346.

47. Frank LD, Glanz K, McCarron M et al. (2006) The spatial distribution of food outlet type and quality around schools in differing built environment and demographic contexts. Berkeley Plann J 19, 79-95.

48. Baker EA, Schootman M, Barnidge E et al. (2006) The role of race and poverty in access to foods that enable individuals to adhere to dietary guidelines. Prev Chronic Dis 3, A76.

49. Block D \& Kouba J (2006) A comparison of the availability and affordability of a market basket in two communities in the Chicago area. Public Health Nutr 9, 837-845.

50. Owen N, Humpel N, Leslie E et al. (2004) Understanding environmental influences on walking; review and research agenda. Am J Prev Med 27, 67-76.

51. Sharkey JR \& Horel S (2008) Neighborhood socioeconomic deprivation and minority composition are associated with better potential spatial access to the ground-truthed food environment in a large rural area. J Nutr 138, 620-627.

52. Frank LD, Andresen MA \& Schmid TL (2004) Obesity relationships with community design, physical activity, and time spent in cars. Am J Prev Med 27, 87-96.

53. Bailey TC \& Gatrell AC (1995) Interactive Spatial Data Analysis. Reading, MA: Addison-Wesley Publishers.

54. Banos A \& Huguenin-Richard F (2000) Spatial distribution of road accidents in the vicinity of point sources: application to child pedestrian accidents. In Geography and Medicine, pp. 54-64 [A Flahault and L Toubiana, editors]. Paris: Elsevier.

55. Maroko AR, Maantay JA, Sohler NL et al. (2009) The complexities of measuring access to parks and physical activity sites in New York City: a quantitative and qualitative approach. Int J Health Geogr 8, 34 .

56. Portnov B, Dubnov J \& Barchana M (2009) Studying the association between air pollution and lung cancer incidence in a large metropolitan area using a kernel density function. Socioecon Plann Sci 43, 141-150.

57. Kloog I, Haim A \& Portnov B (2009) Using kernel density function as an urban analysis tool: investigating the association between nightlight exposure and the incidence of breast cancer in Haifa, Israel. Comput, Environ Urban Syst 33, 55-63.

58. Guagliardo M (2004) Spatial accessibility of primary care: concepts, methods and challenges. Int J Health Geogr 3.

59. Ozdenerol E, Williams BL, Kang SY et al. (2005) Comparison of spatial scan statistic and spatial filtering in estimating low birth weight clusters. Int J Health Geogr 4, 19.

60. Alves de Souza E, Da Silva-Nunes M, Dos Santos Malafronte R et al. (2007) Prevalence and spatial distribution of intestinal parasitic infections in a rural Amazionian settlement, Acre State, Brazil. Cad Saude Publica 23, 427-434.

61. Kulldorff M \& Nagawalla N (1995) Spatial disease clusters: detection and inference. Stat Med 14, 799-819.

62. Apparicio P, Abdelmajid M, Riva M et al. (2008) Comparing alternative approaches to measuring the geographical accessibility of urban health services: distance types and aggregation-error issues. Int J Health Geogr 7, 7.

63. Zenk SN, Schulz AJ, Israel BA et al. (2005) Neighborhood racial composition, neighborhood poverty, and the spatial accessibility of supermarkets in metropolitan Detroit. Am J Public Health 95, 660-667.

64. Beaulac J, Kristjansson E \& Cummins S (2009) A systematic review of food deserts, 1966-2007. Prev Chronic Dis 6, A105. 
65. Chaix B, Merlo J \& Chauvin P (2005) Comparison of a spatial approach with the multilevel approach for investigating place effects on health: the example of healthcare utilisation in France. J Epidemiol Community Health 59, $517-526$

66. Chaix B, Merlo J, Subramanian SV et al. (2005) Comparison of a spatial perspective with the multilevel analytical approach in neighborhood studies: the case of mental and behavioral disorders due to psychoactive substance use in Malmo, Sweden, 2001. Am J Epidemiol 162, 171-182.

67. Matthews SA, Detwiler JE \& Burton LM (2005) Geoethnography: coupling geographic information techniques with ethnographic methods in urban research. Cartographica 40, 75-90.

68. Spielman SE \& Yoo EH (2009) The spatial dimensions of neighborhood effects. Soc Sci Med 68, 1098-1105.

69. Cummins S, Curtis S, Diez-Roux AV et al. (2007) Understanding and representing 'place' in health research: a relational approach. Soc Sci Med 65, 1825-1838.

70. Chaix B, Merlo J, Evans D et al. (2009) Neighbourhoods in eco-epidemiologic research: delimiting personal exposure areas. A response to Riva, Gauvin, Apparicio and Brodeur. Soc Sci Med 69, 1306-1310.

71. Brownson RC, Hoehner CM, Day K et al. (2009) Measuring the built environment for physical activity: state of the science. Am J Prev Med 36, S99-S123.

72. Lovett A, Haynes R, Sunnenberg G et al. (2002) Car travel time and accessibility by bus to general practitioner services: a study using patient registers and GIS. Soc Sci Med 55, 97-111.

73. Martin D, Wrigley H, Barnett $S$ et al. (2002) Increasing the sophistication of access measurement in a rural healthcare study. Health Place 8, 3-13.

74. Burgoine T, Lake AA, Stamp E et al. (2009) Changing foodscapes 1980-2000, using the ASH30 study. Appetite 53, $157-165$.
75. Boone JE, Gordon-Larsen P, Stewart JD et al. (2008) Validation of a GIS facilities database: quantification and implications of error. Ann Epidemiol 18, 371-377.

76. Porter DE, Kirtland KA, Neet MJ et al. (2004) Considerations for using a geographic information system to assess environmental supports for physical activity. Prev Chronic Dis 1, A20.

77. Gould P (1969) Spatial Diffusion. Commission on College Geography Resource Paper no. 4. Washington, DC: Association of American Geographers.

78. Penchansky R \& William Thomas J (1981) The concept of access. Definition and relationship to consumer satisfaction. Med Care XIX, 127-140.

79. Kamphuis CB, van Lenthe FJ, Giskes $\mathrm{K}$ et al. (2007) Perceived environmental determinants of physical activity and fruit and vegetable consumption among high and low socioeconomic groups in the Netherlands. Health Place 13, 493-503.

80. Banos A \& Banos F (2003). Towards integrated surveillance tools of urban risks: space-time analysis of road accidents in Lille. In Géographie des risques des transports, pp. 9-22 [A Banos, F Banos, T Brossard et al., editors]. Orléans: Paradigme.

81. Anselin L, Syabri I \& Kho Y (2006) GeoDa: an introduction to spatial data analysis. Geogr Anal 38, 5-22.

82. Gatrell A \& Naumann I (1992) Hospital location planning: a pilot GIS study. In Proceedings of the Mapping Awareness Conference, pp. 257-258. Birmingham: Miles Arnold.

83. Cummins S (2007) Neighbourhood food environment and diet - time for improved conceptual models? Prev Med $\mathbf{4 4}$, 196-197.

84. Weber C \& Hirsch J (1999) Potential model application and planning issues. Presented at 11th European Colloquium on Quantitative and Theoretical Geography, Cybergeo (article 132), Durham Castle, UK, 3-7 September. 